\title{
Expression of microRNAs in the serum exosomes of methamphetamine-dependent rats vs. ketamine-dependent rats
}

\author{
HANCHENG LI* , CHAN LI* ${ }^{*}$ YUTING ZHOU* ${ }^{*}$ CHAOHUA LUO, JINGYING OU, JING LI and ZHIXIAN MO \\ Department of Pharmacology of Traditional Chinese Medicine, School of Traditional Chinese Medicine, \\ Southern Medical University, Guangzhou, Guangdong 510515, P.R. China
}

Received August 27, 2017; Accepted January 5, 2018

DOI: $10.3892 /$ etm.2018.5814

\begin{abstract}
Drug abuse is a public health and social problem. A number of studies have reported that drug addiction is associated with microRNAs (miRNAs). By comparing the expression of miRNAs in the serum exosomes of methamphetamine-dependent and ketamine-dependent rats, the aim of the present study was to provide insights into the miRNA-mediated associations between the two groups. Published results on conditioned place preference (CPP) in rats conditioned by methamphetamine and ketamine were replicated. The expression of miRNAs in serum exosomes were determined by gene-chip sequencing. The potential target genes of differentially expressed (DE) co-miRNAs were predicted in the methamphetamine and ketamine rats, then functional analysis of their target genes was performed. Methamphetamine and ketamine reward greatly increased the activity time and distance in the intrinsically non-preferred side of the behavioral apparatus when compared with controlled rats $(\mathrm{P}<0.01)$. In addition, methamphetamine upregulated the expression of 276 miRNAs and downregulated 25 miRNAs, while ketamine only downregulated the expression of 267 miRNAs. Ten DE co-miRNAs in the two model groups were identified. Functional analysis revealed that DE co-miRNAs are involved in the development of addiction at different stages, and their target genes were enriched in 'vesicular transport', 'amphetamine addiction', 'dopaminergic synapse' and 'GABAergic synapse'. Therefore, it was suggested that these co-miRNAs may have a strong association with drug addiction, and they may be involved in the different addiction processes, which partly explains methamphetamine and ketamine addiction.
\end{abstract}

Correspondence to: Professor Zhixian Mo, Department of Pharmacology of Traditional Chinese Medicine, School of Traditional Chinese Medicine, Southern Medical University, 1063 Shatai Road, Guangzhou, Guangdong 510515, P.R. China E-mail: cherrymo001@126.com

*Contributed equally

Key words: addiction, exosomes, microRNAs, methamphetamine, ketamine, functional analysis

\section{Introduction}

Drug abuse has been a public health and social problem. According to the survey, nearly $80 \%$ of drug users are abused of new synthetic drugs (1). Compared with the traditional drugs, the new synthetic drug produced long-lasting and severe damage to the central nervous system (CNS) and more easily leaded to addiction (2). Methamphetamine and ketamine are the most popular new synthetic drugs in recent years. With a chemical structure of CNS stimulants, methamphetamine has a strong central excitatory effect and will produce a mental dependence response after long-term use (3). Ketamine, commonly known as ' $\mathrm{K}$ powder', is a new synthetic drug which is abused worldwide (4). However, the mechanism of ketamine addiction is poorly understood.

At present, CPP test is one of the most effective methods for the determination of animal reward (5). It is a simple but effective means of evaluating drug dependence potential and looking for anti-addiction drugs (6). The essence of drug addiction is a pathological memory based on drug-induced gene expression and changes in synaptic plasticity (7). In recent years, the important biological functions of post-transcriptional regulation have caught widespread attention and extensive research, such as the role of miRNA in drug addiction. Many studies have reported that drug addiction, such as cocaine addiction, nicotine addiction, opioid addiction and alcohol addiction are associated with miRNAs (8-11). Research into the regulatory role played by miRNAs in methamphetamine and ketamine addiction is in a preliminary stage.

Studies have shown that some miRNAs are encapsulated in exosomes which can be picked up by target cells. In this situation, these miRNAs act as the type of endogenous miRNA that silences target genes (12). As a molecular medium, exosomes can transmit information between cells to cause disease, and can carry a variety of substances including protein, mRNA, miRNA, DNA, and lipid (13). Moreover, exosomes are involved in processes of cell migration, cell communication, angiogenesis, tumor-cell growth and tumor immunity (14-16). Therefore, our study replicated a CPP test of methamphetamine and ketamine dependence in rats. Furthermore, by comparing the expression of miRNAs in serum exosomes of methamphetamine and ketamine-dependence rats, this study aims to provide insights into the miRNA-mediated relationship between the two model groups. A total of 10 differentially 
expressed (DE) co-miRNAs both in methamphetamine and ketamine group were further analyzed by bioinformatics.

\section{Materials and methods}

Animals. Sprague-Dawley rats (weight, 170-210 g; age, 2 months) were provided by the Southern Medical University's Experimental Animal Center (no. SCXK GD 2011-0015; Guangzhou, China). All animal experimental protocols and operating procedures were in accordance with the National Institutes of Health (NIH; Bethesda, MD, USA) guidelines for the experimental use of laboratory animals. The experiments were approved by the Experimental Animal Ethics Committee of Southern Medical University.

Reagents and drugs. Ketamine Hydrochloride Injection (no. 1311-7603) was purchased from Fujian Gutian Pharmaceutical Co., Ltd. (Fujian, China). Methamphetamine (no. 1212-9802) was obtained from the National Narcotics Laboratory (Beijing, China). The TruSeq ${ }^{\circledR}$ Small RNA Sample Prep kit (no. 4472908) was purchased from Illumina Corporation. The Qubit ${ }^{\circledR}$ dsDNA HS Assay kit (no. 4367809) was purchased from Thermo Fisher Scientific, Inc. (Waltham, MA, USA). U6 was used as the calibration gene to provide an internal control. Primers were synthesized by the Guangzhou Ruibo Biotechnology Co., Ltd. (Guangzhou, China). All other chemicals used in these experiments were of analytical grade.

Apparatus. The CPP apparatus consisted of two equal-sized compartments (30 cm long x $30 \mathrm{~cm}$ wide x $30 \mathrm{~cm}$ high), including one having a white interior and the other having a black interior that separated by a wall with a sliding door. Bio-Rad Gradient PCR (Bio-Rad Laboratories, Inc., Hercules, CA, USA); Agilent 2200 Bioanalyzer; Stratagene Mx3005P Real-time polymerase chain reaction (PCR) instrument (Agilent Technologies, Inc., Santa Clara, CA, USA); Agilent 2200 TapeStation (Agilent Technologies, Inc.); DU800 Nucleic Acid/Protein Analyzer (Beckman Coulter, Inc., Brea, CA, USA); IX53 Fluorescence Inverted Microscope (Olympus Corporation, Tokyo, Japan); Qubit 2.0 (Thermo Fisher Scientific, Inc.); Hiseq 2500 (Illumina, Inc., San Diego, CA, USA) and ND-1000 Nanodrop (Thermo Fisher Scientific, Inc., Wilmington, DE, USA).

Conditioned place preference (CPP) paradigm. Rats were tested in a CPP apparatus as described previously. Suitable rats were randomly divided into three groups of ten: Control group, methamphetamine group and ketamine group. The method of the CPP paradigm was copied from our previous reports $(17,18)$ and training process for CPP test is shown in Fig. 1.

RNA extraction and gene-chip sequencing. The extraction of RNA from the exosome was performed in accordance with QIAGEN's miRNeasy Mini lit manual. The TRIzol method was used for sample extraction and samples were tested with the Agilent 2200 Tape station system and the Nano drop ND-1000 Spectrophotometer. Total RNA (initial volume $1 \mu \mathrm{l})$ from each acceptable sample was used to construct a miRNA library using the TruSeq ${ }^{\circledR}$ Small RNA Sample Prep kit (Illumina, Inc.). Then the library of acceptable miRNAs

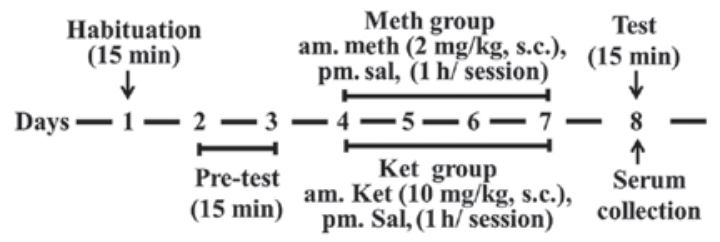

Figure 1. Training process for conditioned place preference test. Meth, methamphetamine; Ket, ketamine; Sal, physiological saline.

was submitted to sample preparation according to the method described in the lllumina HiSeq 2500 User Guide, the final concentration of sample being $10 \mathrm{pM}$.

Validation of selected DE miRNAs by reverse transcription-quantitative PCR (RT-qPCR). Then, $1 \mu 1$ total RNA from each sample was taken and synthesized into cDNA by reverse transcription according to the manual of the reverse transcription kit. One microliter of reverse-transcribed cDNA was taken and evaluated according to the instructions for $\mathrm{SYBR}^{\circledR}$ Select Master Mix dye by Real-Time PCR. The 7900HT Fast Real-Time PCR System was used to amplify the different genes. The program used for amplification was as follows: $2 \mathrm{~min}$ at $50^{\circ} \mathrm{C}, 2 \mathrm{~min}$ at $95^{\circ} \mathrm{C}$, followed by 40 cycles of $15 \mathrm{sec}$ at $95^{\circ} \mathrm{C}$ for denaturation, and $1 \mathrm{~min}$ at $60^{\circ} \mathrm{C}$ for annealing and extension. U6 was used as a reference gene. Three independent experiments were performed.

miRNA sequence analysis and functional analysis. The expression of miRNAs in each library was estimated using the free web-server tool sRNAbench (http://seqanswers. com), where the normalized read count of each miRNA was shifted by the following formula: $R P M=($ miRNAs read number/total map reads) x 1,000,000. Evaluation of DE of miRNAs was employed to focus our sugar-edge study (http://pubs.acs.org/doi/abs/10.1021/jp053379q). miRNAs were characterized by having $\log _{2} \mathrm{FC}>1$ or $<-1, \mathrm{P}<0.01$ (19). Target Scan (http://targetscan.org/), DIANA Tools (http://diana.imis.athena-innovation.gr/DianaTools/), and miRDB (http://www.mirdb.org/cgi-bin/search.cgi) were used to predict the target genes of selected DE miRNAs. GO (Gene Ontology) and KEGG (Kyoto Encyclopedia of Genomes and Genes), accessed on the David site (http://david.abcc.ncifcrf. gov). The top $10 \mathrm{GO}$ terms and KEGG pathways were identified. A threshold of $\mathrm{P}<0.05$ was used to enrich each miRNA's significant biological functions. The net map of protein-protein interaction (PPI) was analyzed by STRING online analysis software (https://string-db.org/).

Statistical analysis. Data were expressed as mean \pm SD and analyzed by using one-way analysis of variance, followed by the two-tailed, least-significant-difference post hoc test. Statistical analyses were performed using SPSS software (version 17.0; SPSS, Inc., Chicago, IL, USA). P $<0.05$ was considered to indicate a statistically significant difference.

\section{Results}

The behavioral alteration induced by methamphetamine and ketamine. The effects of methamphetamine and ketamine 
Table I. Time span and exercise distance of rats in the white compartment following injections of methamphetamine and ketamine.

\begin{tabular}{|c|c|c|c|c|}
\hline \multirow[b]{2}{*}{ Group } & \multicolumn{2}{|c|}{ Activity time in white side ( $\mathrm{sec}$ ) } & \multicolumn{2}{|c|}{ Activity distance in white side $(\mathrm{cm})$} \\
\hline & Pre-CPP & Post-CPP & Pre-CPP & Post-CPP \\
\hline Control & $192.93 \pm 22.89$ & $202.67 \pm 28.24$ & $3,182.89 \pm 323.22$ & $3,349.26 \pm 412.28$ \\
\hline Methamphetamine & $201.47 \pm 25.98$ & $460.47 \pm 66.71^{\mathrm{a}}$ & $3,242.86 \pm 308.59$ & $4,475.97 \pm 585.74^{\mathrm{a}}$ \\
\hline Ketamine & $195.11 \pm 19.32$ & $435.09 \pm 50.34^{\mathrm{a}}$ & $3,123.37 \pm 317.30$ & $4,501.02 \pm 577.21^{\mathrm{a}}$ \\
\hline F-value & 0.910 & 63.462 & 0.243 & 58.732 \\
\hline P-value & 0.532 & $1.715 \times 10^{-5}$ & 0.668 & $2.303 \times 10^{-4}$ \\
\hline
\end{tabular}

Data are expressed as mean \pm standard deviation $(n=10) .{ }^{a} \mathrm{P}<0.01$ vs. control group. $\mathrm{CPP}$, conditioned place preference.

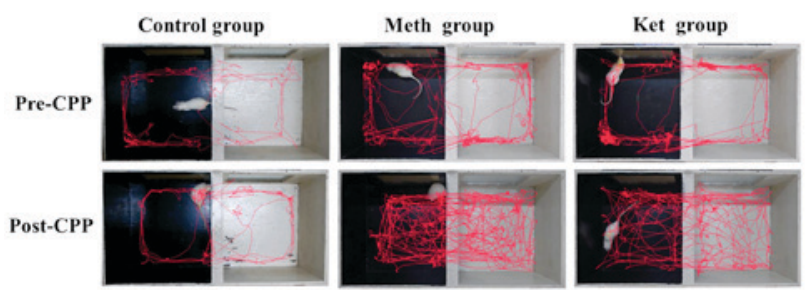

Figure 2. Activity routes of rats in the CPP compartment. Meth, methamphetamine; Ket, ketamine; CPP, conditioned place preference.

on CPP rats were shown in Table I. Both methamphetamine and ketamine greatly increased the activity time and activity distance in the non-preferred (white) compartment compared with that of the control rats $(\mathrm{P}<0.01)$. Rat activity routes in CPP are shown in Fig. 2. In comparison with those of the control rats, the activity routes in the non-preferred compartment were increased greatly by methamphetamine and ketamine.

The alteration of miRNAs expression induced by methamphetamine and ketamine. Compared with control group, there were 301 miRNAs DE in methamphetamine group. Among them, there were 276 miRNAs high expressed after methamphetamine induction, and 25 were low expressed (Fig. 3A). Among 276 high expressed miRNAs, there are 50 miRNAs were significantly $\mathrm{DE}(\log 2 \mathrm{FC}>1, \mathrm{P}<0.01)$, accounting for $96.15 \%$ of the number of significant DE miRNAs. Among 25 low expressed miRNAs, there are two miRNAs were significantly DE $(\log 2 \mathrm{FC}<-1, \mathrm{P}<0.01)$, accounting for $3.85 \%$ of the number of significant DE miRNAs. A heat map of 52 (50 high expressed and two low expressed) significantly DE miRNAs in serum exosomes of methamphetamine rats showed in Fig. 3B.

Compared with control group, there were 267 miRNAs DE in ketamine group (Fig. 3C). Among them, there were all low expressed after ketamine induction, and 122 miRNAs were $\mathrm{DE}(\log 2 \mathrm{FC}<-1, \mathrm{P}<0.01)$. A heat map of $122 \mathrm{DE}$ miRNAs in serum exosomes of ketamine rats showed in Fig. 3D. Further analyzed of 52 DE miRNAs in methamphetamine group and 122 DE miRNAs in ketamine group by venn diagram, we found that ten miRNAs were DE co-miRNAs (Table II). Most of these miRNAs were up-regulated by methamphetamine, but down-regulated by ketamine.
Functional analysis. Based on the above analysis, we selected these ten DE co-miRNAs for target gene prediction. A total of 1151 targets of these miRNAs were predicted by TargetScan, miRDB, and DIANA tools. Table III gives more information on these miRNAs. These predicted targets were classed into 633 kinds according to the biological processes of the GO categories $(\mathrm{P}<0.05)$, specially including nervous system development, neuron generation and differentiation, apoptotic process as well as cell death (Fig. 4A). For KEGG pathway analysis, there were $26 \mathrm{KEGG}$ pathways have statistically significant $(\mathrm{P}<0.05)$, specially including SNARE interactions in vesicular transport, amphetamine addiction, cGMP-PKG signaling pathway, dopaminergic synapse and GABAergic synapse. The above results suggested that the DE miRNAs and their target genes are related to vesicle transport, amphetamine addiction and neurotransmitter delivery (Fig. 4B).

Proteins-proteins interactions (PPI) analysis. A total of 1,151 targets of these ten DE miRNAs were analyzed by STRING software, and the PPI net map was showed in Fig. 5. The PPI shows a complex interaction of target genes encoding proteins. STX5, BET1, CLTB, CLTC, ACLY, ENO2, TNFRSF1B, SNAP23, VAMP2, STX6 and VAMP4 have core positions in PPI network.

Validation of miRNAs by RT-qPCR. To further verify the accuracy of our gene-chip analysis, we selected four DE miRNAs from these ten DE co-miRNAs of methamphetamine group for verification by RT-qPCR. The dissolution-curve analysis showed no heterozygotes, indicating that the amplification products were single and free from nonspecific amplification. The amplification curve indicated that all samples had entered the expansion platform period, indicating that the reaction conditions had been set accurately. From Fig. 6, we see that the RT-qPCR results are consistent with those of gene-chip sequencing.

\section{Discussion}

The CPP test is the classic experiment to detect psychological drug dependence (20). Our results showed that methamphetamine and ketamine significantly increased the activity time and distance of rats in the non-preferred compartment after CPP, which suggest that we have replicated the CPP test successfully. From the difference analysis of miRNA 
Table II. Ten differentially expressed co-miRNAs in the methamphetamine and ketamine groups.

\begin{tabular}{|c|c|c|c|c|}
\hline \multirow[b]{2}{*}{ miRNA_ID } & \multicolumn{2}{|c|}{ Control vs. Meth } & \multicolumn{2}{|c|}{ Control vs. Ket } \\
\hline & $\log 2$ (fold-change) & P-value & $\log 2$ (fold-change) & P-value \\
\hline rno-miR-128-3p & -1.3445 & $5.83 \times 10^{-4}$ & -4.6258 & $5.79 \times 10^{-5}$ \\
\hline rno-miR-133a-3p & 4.7943 & $3.51 \times 10^{-7}$ & -7.4342 & $1.98 \times 10^{-2}$ \\
\hline rno-miR-152-3p & 2.5283 & $2.52 \times 10^{-2}$ & -4.3439 & $2.88 \times 10^{-4}$ \\
\hline rno-miR-181a-5p & 2.6260 & $1.77 \times 10^{-2}$ & -4.7517 & $3.51 \times 10^{-5}$ \\
\hline rno-miR-192-3p & 6.9336 & $6.15 \times 10^{-3}$ & -7.4342 & $1.98 \times 10^{-2}$ \\
\hline rno-miR-194-5p & 4.7535 & $9.42 \times 10^{-7}$ & -5.3395 & $2.23 \times 10^{-6}$ \\
\hline rno-miR-218b & 2.6077 & $2.35 \times 10^{-2}$ & -3.6964 & $1.07 \times 10^{-2}$ \\
\hline rno-miR-22-5p & 2.7264 & $1.72 \times 10^{-2}$ & -3.5319 & $1.31 \times 10^{-2}$ \\
\hline rno-miR-362-3p & 2.8471 & $3.30 \times 10^{-2}$ & -3.9071 & $4.44 \times 10^{-3}$ \\
\hline rno-miR-674-3p & 3.1519 & $1.13 \times 10^{-2}$ & -10.9521 & $9.89 \times 10^{-9}$ \\
\hline
\end{tabular}

miR, microRNA; rno, Rattus norvegicus; Meth, methamphetamine; Ket, ketamine.

A

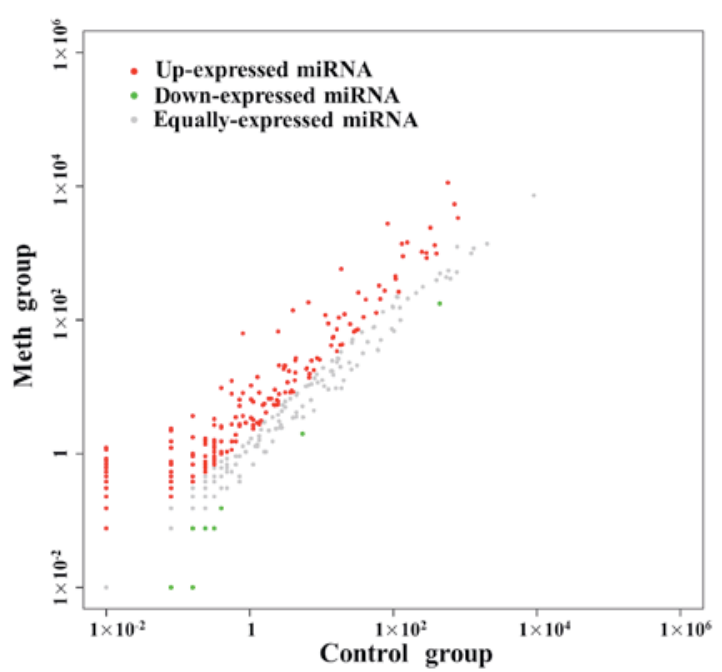

C

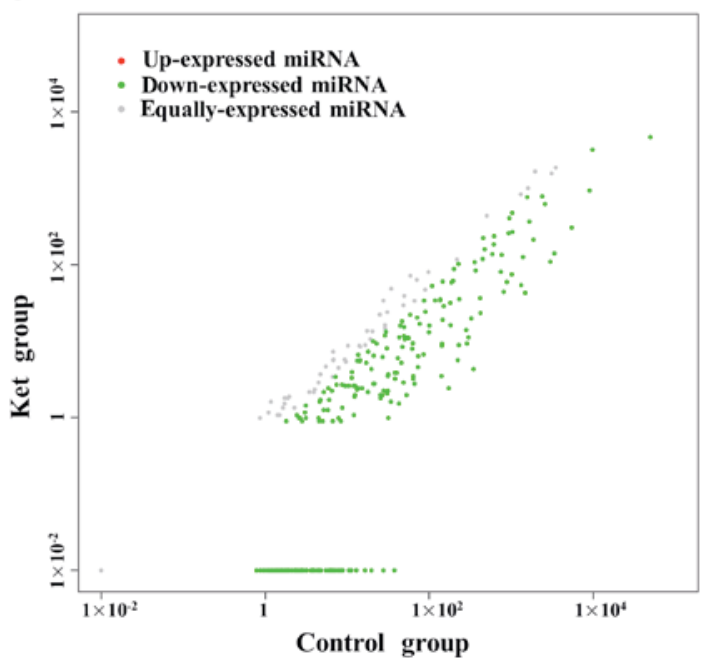

B

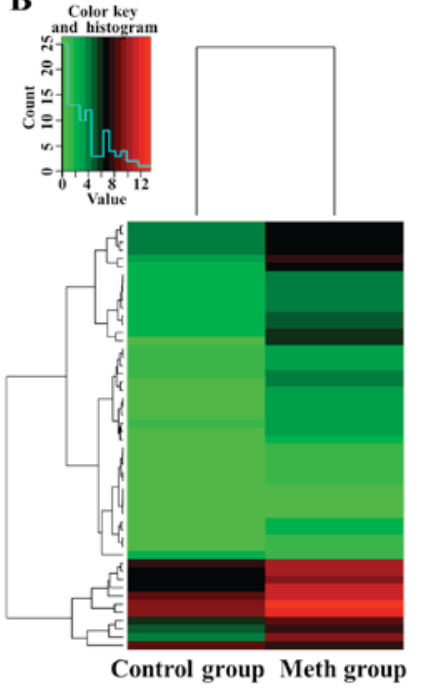

D

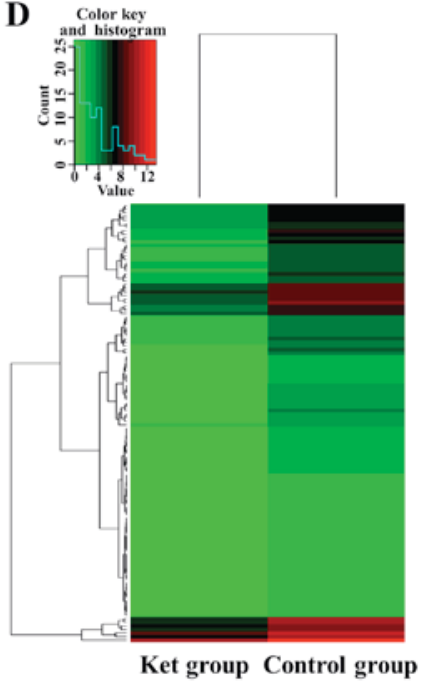

Figure 3. Scatter plots and heat maps of differentially expressed miRNAs in the serum exosomes of the methamphetamine and ketamine groups. (A) A scatter plot map showing 301 differentially expressed miRNAs in the methamphetamine group. (B) A heat map presenting 52 differentially expressed miRNAs in the methamphetamine group. (C) A scatter plot map showing 267 differentially expressed miRNAs in the ketamine group. (D) A heat map exhibiting 122 differentially expressed miRNAs in the ketamine group. miRNA, microRNA; Meth, methamphetamine; Ket, ketamine. 
Table III. Information on selected microRNAs and the number of candidate genes.

\begin{tabular}{lllr}
\hline miRNA ID & Accession number & \multicolumn{1}{c}{ miRNA sequence } & No. candidate genes \\
\hline rno-miR-128-3p & MIMAT0000834 & UCACAGUGAACCGGUCUCUUU & 106 \\
rno-miR-133a-3p & MIMAT0000839 & UUUGGUCCCCUUCAACCAGCUG & 57 \\
rno-miR-152-3p & MIMAT0000854 & UCAGUGCAUGACAGAACUUGG & 177 \\
rno-miR-192-3p & MIMAT0017147 & CUGCCAGUUCCAUAGGUCACAG & 133 \\
rno-miR-218b & MIMAT0017838 & CAUGGUUAGAUCAAGCACAA & 68 \\
rno-miR-22-5p & MIMAT0003152 & AGUUCUUCAGUGGCAAGCUUUA & 66 \\
rno-miR-362-3p & MIMAT0017357 & AACACACCUGUUCAAGGAUUCA & \\
rno-miR-674-3p & MIMAT0005330 & CACAGCUCCCAUCUCAGAACAA & 63 \\
rno-miR-194-5p & MIMAT0000869 & UGUAACAGCAACUCCAUGUGGA & 53 \\
rno-miR-181a-5p & MIMAT0000858 & AACAUUCAACGCUGUCGGUGAGU & 108 \\
\hline
\end{tabular}

miR/miRNA, microRNA.

A

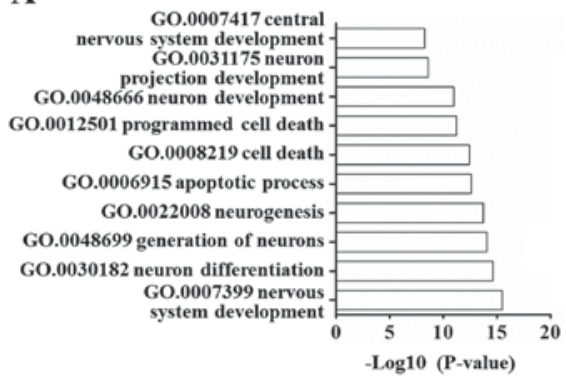

B

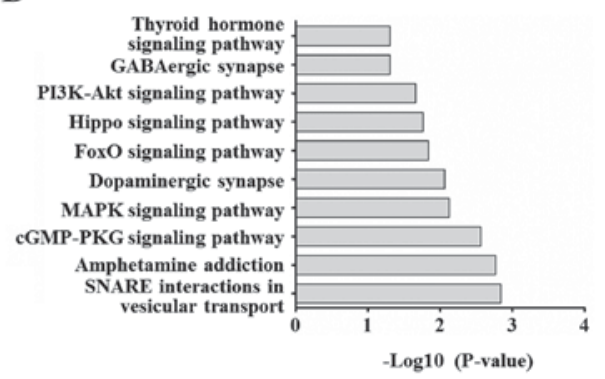

Figure 4. GO and KEGG pathway analyses of the selected DE miRNAs' targets. (A) Top 10 sorts enriched GO terms of the selected DE miRNAs' targets. (B) Top 10 sorts enriched KEGG pathways of the selected DE miRNAs' targets. The y-axes represent the GO or KEGG terms, respectively, and the x-axes indicate the values presented as $-\log _{10}\left(\mathrm{P}\right.$-value); the smaller the P-value, the larger the value of - $\log _{10}(\mathrm{P}$-value). GO, gene ontology; KEGG, Kyoto Encyclopedia of Genes and Genomes; miRNA, microRNA; DE, differentially expressed.

expression, methamphetamine could up regulate the expression of most miRNA, and ketamine down regulate the expression of miRNA. These suggest that different addictive drugs have different effects on the expression of miRNA in rats.

There are ten DE miRNAs both in the two model groups, such as miR-128-3p, miR-133a-3p, miR-152-3p and miR-181a-5p. By GO analysis, we found that the biological processes of these ten miRNAs target genes mainly enriched in nervous system development, neuron generation and differentiation, apoptotic process as well as cell death. The essence of drug addiction is a pathological memory based on drug-induced gene expression and changes in synaptic plasticity (21).

From KEGG pathway analysis, we also found that these target genes mainly located in SNARE interactions in vesicular transport, amphetamine addiction, cGMP-PKG signaling pathway, dopaminergic synapse and GABAergic synapse. Many of these pathways involved in drug addiction. In our study, these ten DE miRNAs were all from serum exosome. Exosome is a nano-sized vesicular body (22). It can transport miRNA to target organs or cells, so pathway of SNARE interactions in vesicular transport may related to DE co-miRNAs transportion and then involved in drug addiction. The mesolimbic dopamine system is involved in the rewarding effects of almost all addictive drugs (23). Central nervous stimulants increased the release of dopamine and the concentration of dopamine in synaptic gap, and then caused addictive behavior (24). GABA energy system is the most important inhibitory system in the CNS (25). It has been found that the withdrawal of morphine addiction leads to an increase in the inhibitory effect of GABA on systemic (26), suggesting that morphine addiction is closely related to the GABA system.

The PPI shows a complex interaction of target genes encoding proteins. Many target genes are related to vesicle transport, neurons protection and apoptosis. As the main structural component of the lattice-type cytoplasmic face of coated pits and vesicles, CLTB entrap specific macromolecules during receptor-mediated endocytosis (27). SNAP23 is an essential component of the high affinity receptor for the general membrane fusion machinery and is an important regulator of transport vesicle docking and fusion (28). In nervous tissue, ACLY may be involved in the biosynthesis of acetylcholine (29). The activity of cholinergic transmitters in the brain is closely related to cognitive processes. Knockout studies in mice also suggest a role of TNFRSF1B in protecting neurons from apoptosis by stimulating antioxidative pathways (30). RAD21 plays a role in apoptosis, via its cleavage by caspase-3/CASP3 or caspase-7/CASP7 during early steps of apoptosis (31).

In this study, methamphetamine significantly increased the activity time and distance of rats in the non-preferred 


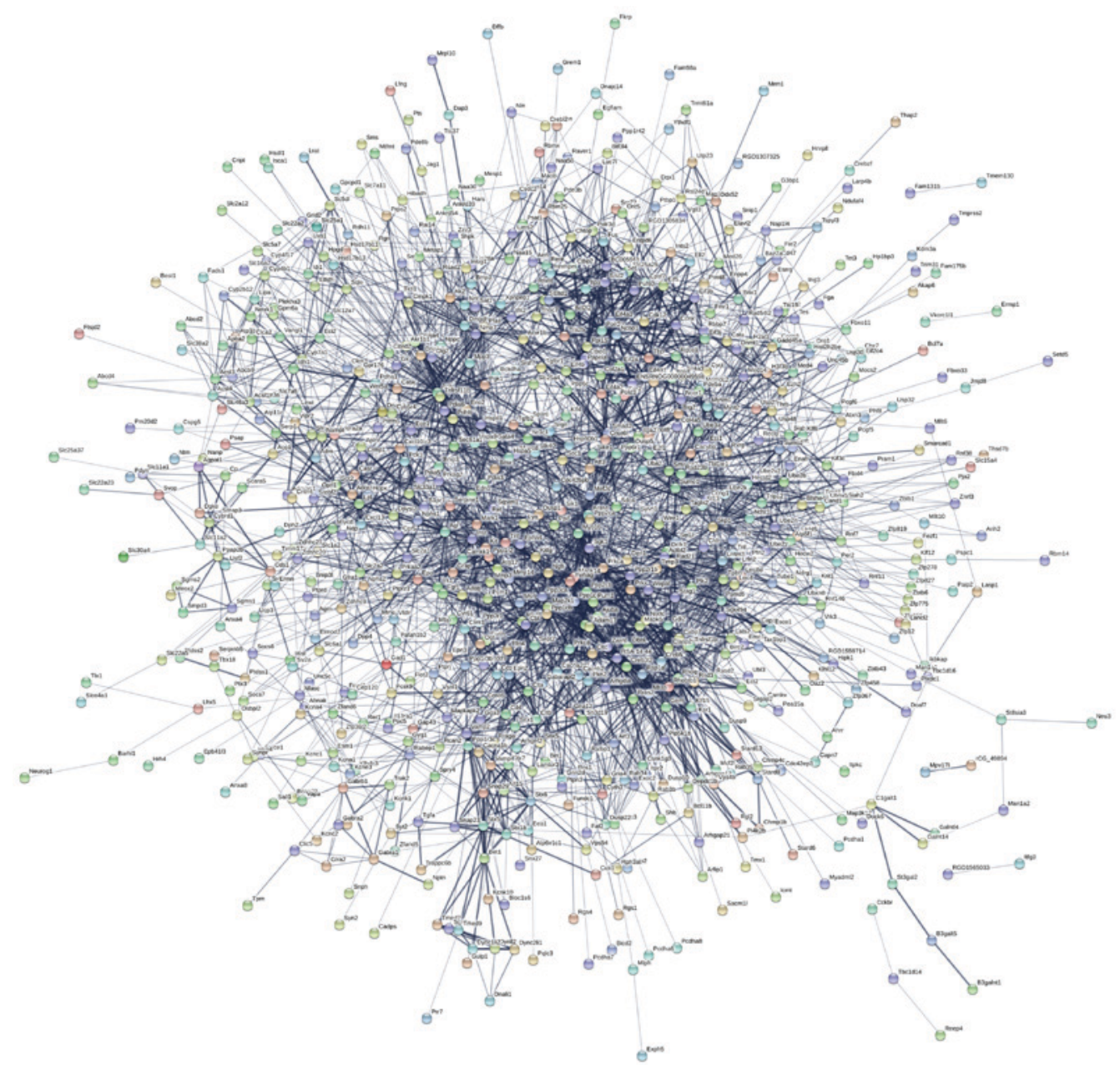

Figure 5. Protein-protein interaction net map of 10 differentially expressed co-microRNAs.

A

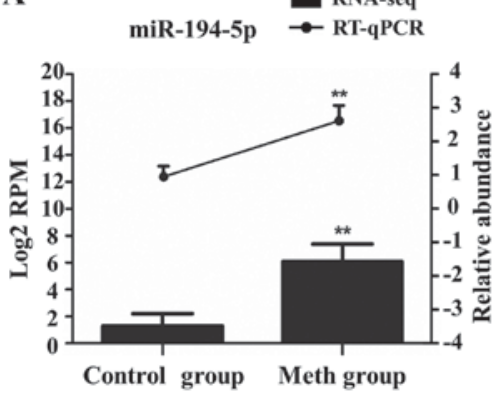

C

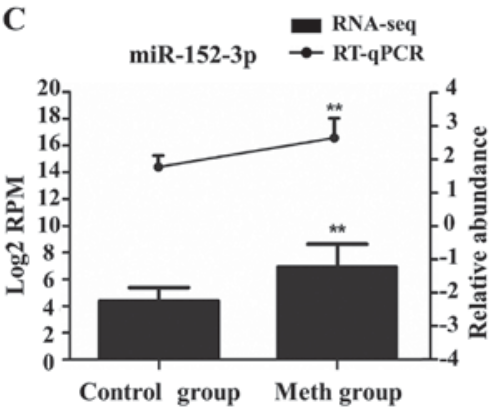

miR-22-3p $\rightarrow$ RNA-seq

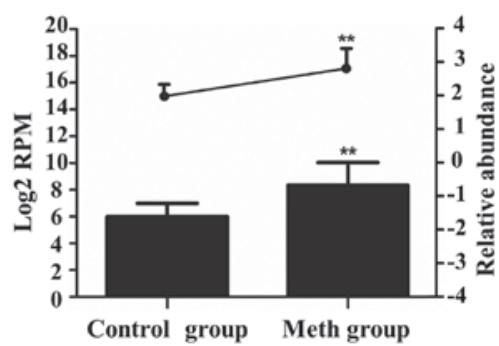

D

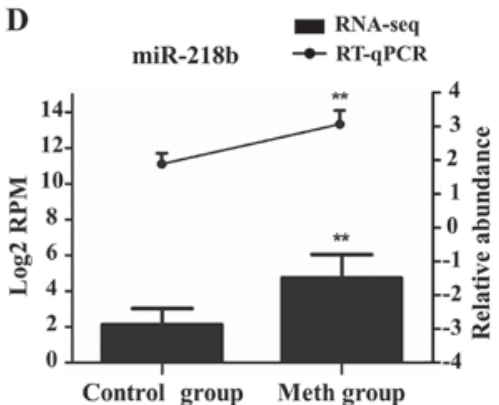

Figure 6. miRNA expression in the methamphetamine group. (A) miR-194-5p, (B) miR-22-3p, (C) miR-152-3p and (D) miR-218b in the methamphetamine group were detected by RT-qPCR and miRNA-seq. miRNA expression from RT-qPCR is represented by lines at the top of each graph; the values are shown on the right $\mathrm{y}$-axis as the relative abundance. miRNA expression data from miRNA-seq is presented as a bar chart; the values are shown on the left y-axis as $\log _{2}$ RPM (normalized reads number). Data are presented as the mean \pm standard deviation. ${ }^{* *} \mathrm{P}<0.01$ vs. control group. miR/miRNA, microRNA; RT-qPCR, reverse transcription-quantitative polymerase chain reaction; miRNA-seq, miRNA sequencing; RPM, reads per million. 
compartment after CPP and up regulated most miRNAs expression. Ketamine could also increase the activity time and distance of rats in the non-preferred compartment after CPP, but down regulated all miRNAs expression. It suggests that the mechanism of methamphetamine and ketamine addiction is different. As to the biological processes and pathways are involved in the development of addiction at multiple stages, we speculate these ten DE miRNAs both in methamphetamine group and ketamine group are closely related to drug addiction. These biological processes of target genes may be common or partly involved in the process of methamphetamine and ketamine addiction, but further studies are necessary. Because of their role in regulating important molecules, our results are important for theory.

\section{Acknowledgements}

This study was supported by National Natural Science Foundation of China (no. 81673628 and 81229003), Guangdong Science and Technology Project (no. 2016A020226003), Natural Science Foundation of Guangdong Province (no. 2014A030310251) and State of traditional Chinese medicine of Guangdong province (no. 20162085 and 20171165).

\section{References}

1. Tomás-Rosselló J, Rawson RA, Zarza MJ, Bellows A, Busse A, Saenz E, Freese T, Shawkey M, Carise D, Ali R and Ling W: United nations office on drugs and crime international network of drug dependence treatment and rehabilitation resource centres: Treatnet. Subst Abus 31: 251-263, 2010.

2. Vinogradova MV,KryukovaEM,KulyaminaOS, Vapnyarskaya OI and Sokolova AP: Approaches to the study of the status and trends of drug abuse, rehabilitation and reintegration of drug users. Biosci Biotechnol Res Asia 11: 1505-1514, 2014.

3. Baptista S, Lourenço J, Milhazes N, Borges F, Silva AP and Bacci A: Long-term treatment with low doses of methamphetamine promotes neuronal differentiation and strengthens long-term potentiation of glutamatergic synapses onto dentate granule neurons. eNeuro 3: pii: ENEURO, 2016.

4. Stoicea N, Versteeg G, Florescu D, Joseph N, Fiorda-Diaz J, Navarrete $\mathrm{V}$ and Bergese SD: Ketamine-based anesthetic protocols and evoked potential monitoring: A risk/benefit overview. Front Neurosci 10: 37, 2016.

5. Tzschentke TM: Measuring reward with the conditioned place preference (CPP) paradigm: Update of the last decade. Addict Biol 12: 227-462, 2007.

6. Tzschentke TM: Measuring reward with the conditioned place preference paradigm: A comprehensive review of drug effects, recent progress and new issues. Prog Neurobiol 56: 613-672, 1998.

7. Sun W: Dopamine neurons in the ventral tegmental area: Drug-induced synaptic plasticity and its role in relapse to drug-seeking behavior. Curr Drug Abuse Rev 4: 270-285, 2011.

8. Hollander JA, Im HI, Amelio AL, Kocerha J, Bali P, Lu Q, Willoughby D, Wahlestedt C, Conkright MD and Kenny PJ: Striatal microRNA controls cocaine intake through CREB signalling. Nature 466: 197-202, 2010.

9. Maccani MA, Avissar-Whiting M, Banister CE, McGonnigal B, Padbury JF and Marsit CJ: Maternal cigarette smoking during pregnancy is associated with downregulation of mir-16, mir-21, and mir-146a in the placenta. Epigenetics 5: 583-589, 2010.

10. Zheng $\mathrm{H}$, Zeng Y, Zhang X, Chu J, Loh HH and Law PY: mu-Opioid receptor agonists differentially regulate the expression of miR-190 and NeuroD. Mol Pharmacol 77: 102-109, 2010.

11. Baek MN, Jung KH, Halder D, Choi MR, Lee BH, Lee BC, Jung MH, Choi IG, Chung MK, Oh DY and Chai YG: Artificial microRNA-based neurokinin-1 receptor gene silencing reduces alcohol consumption in mice. Neurosci Lett 475: 124-128, 2010.

12. Emmanouilidou E, Melachroinou K, Roumeliotis T, Garbis SD, Ntzouni M, Margaritis LH, Stefanis L and Vekrellis K: Cell-produced alpha-synuclein is secreted in a calcium-dependent manner by exosomes and impacts neuronal survival. J Neurosci 30: 6838-6851, 2010.
13. Simons M and Raposo G: Exosomes-vesicular carriers for intercellular communication. Curr Opin Cell Biol 21: 575-581, 2009.

14. Lai R, Yeo R, Tan S, Zhang B, Yin Y, Sze N, Choo A and Lim S: Biochemical potential of MSC exosome. Cytotherap 16: S43, 2014.

15. Sahoo S, Klychko E, Thorne T, Misener S, Schultz KM, Millay M, Ito A, Liu T, Kamide C, Agrawal H, et al: Exosomes from human CD34(+) stem cells mediate their proangiogenic paracrine activity. Circ Res 109: 724-728, 2011.

16. Wang HW, Wang J, Ding F, Callahan K, Bratkowski MA, Butler JS, Nogales E and Ke A: Architecture of the yeast Rrp44 exosome complex suggests routes of RNA recruitment for 3 ' end processing. Proc Natl Acad Sci USA 104: 16844-16849, 2007.

17. Liu W, Peng QX, Lin XL, Luo CH, Jiang MJ, Mo ZX and Yung KK: Effect of rhynchophylline on the expression of p-CREB and sc-Fos in triatum and hippocampal CA1 area of methamphetamine-induced conditioned place preference rats. Fitoterapia 92: 16-22, 2014.

18. Li J, Liu W, Peng Q, Jiang M, Luo C, Guo Y, Liu Y, Fang M and Mo Z: Effect of rhynchophylline on conditioned place preference on expression of NR2B in methamphetamine-dependent mice. Biochem Biophys Res Commun 452: 695-700, 2014.

19. Robinson MD, McCarthy DJ and Smyth GK: EdgeR: A bioconductor package for differential expression analysis of digital gene expression data. Bioinformatics 26: 139-140, 2010.

20. Manzanedo C, Aguilar MA, Rodriguez-Arias M and Miñarro J: Conditioned place preference paradigm can be a mouse model of relapse to opiates. Neurosci Res Commun 28: 23-29, 2001.

21. Van den Oever MC, Spijker S and Smit AB: The synaptic pathology of drug addiction. Adv Exp Med Biol 970: 469-491, 2012.

22. Gonda DD, Akers JC, Kim R, Kalkanis SN, Hochberg FH, Chen CC and Carter BS: Neuro-oncologic applications of exosomes, microvesicles, and other nano-sized extracellular particles. Neurosurgery 72: 501-510, 2013.

23. Yang MH, Jung MS, Lee MJ, Yoo KH, Yook YJ, Park EY, Choi SH, Suh YJ, Kim KW and Park JH: Gene expression profiling of the rewarding effect caused by methamphetamine in the mesolimbic dopamine system. Mol Cells 26: 121-130, 2008.

24. During MJ, Bean AJ and Roth RH: Effects of CNS stimulants on the in vivo release of the colocalized transmitters, dopamine and neurotensin, from rat prefrontal cortex. Neurosci Lett 140: 129-133, 1992.

25. Vaz GC, Bahia AP, de Figueiredo Müller-Ribeiro FC, Xavier CH, Patel KP, Santos RA, Moreira FA, Frézard F and Fontes MA: Cardiovascular and behavioral effects produced by administration of liposome-entrapped GABA into the rat central nervous system. Neuroscience 285: 60-69, 2015.

26. Nugent FS: VTA GABAergic plasticity: An inhibitory synaptic model of drug addiction. Springer, New York, NY, 2011.

27. Borner GH, Antrobus R, Hirst J, Bhumbra GS, Kozik P, Jackson LP, Sahlender DA and Robinson MS: Multivariate proteomic profiling identifies novel accessory proteins of coated vesicles. J Cell Biol 197: 141-160, 2012.

28. Bauer MC, O'Connell DJ, Maj M, Wagner L, Cahill DJ and Linse S: Identification of a high-affinity network of secretagogin-binding proteins involved in vesicle secretion. Mol Biosyst 7: 2196-2204, 2011

29. Byrne FL, Poon IK, Modesitt SC, Tomsig JL, Chow JD, Healy ME, Baker WD, Atkins KA, Lancaster JM, Marchion DC, et al: Metabolic vulnerabilities in endometrial cancer. Cancer Res 74: 5832-5845, 2014.

30. Surhone LM, Tennoe MT and Henssonow SF: Tnfrsf $1 b$. Betascript Publishing, 2011

31. Pati D, Zhang N and Plon SE: Linking sister chromatid cohesion and apoptosis: Role of Rad21. Mol Cell Biol 22: 8267-8277, 2002.

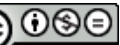

This work is licensed under a Creative Commons Attribution-NonCommercial-NoDerivatives 4.0 International (CC BY-NC-ND 4.0) License. 